\title{
Preliminary clinical assessment of the relationship between tumor alphavbeta3 integrin and perfusion in patients studied with $\left[{ }^{18} \mathrm{~F}\right]$ fluciclatide kinetics and $\left[{ }^{15} \mathrm{O}\right] \mathrm{H}_{2} \mathrm{O}$ PET
}

Laura M Kenny ${ }^{1,2^{*}}$, Giampaolo Tomasi ${ }^{1}$, Federico Turkheimer ${ }^{3}$, James Larkin ${ }^{4}$, Martin Gore ${ }^{4}$, Cathryn S Brock ${ }^{2}$, Stephen Mangar ${ }^{2}$ and Eric O Aboagye ${ }^{1}$

\begin{abstract}
Background: $\left[{ }^{18} \mathrm{~F}\right]$ fluciclatide, a peptide ligand with high affinity for av $\beta 3 / a v \beta 5$ integrins, is a proposed biomarker of tumor angiogenesis. The study rationale was to perform a preliminary evaluation of the relationship between tumor $\left[{ }^{18} \mathrm{~F}\right]$ fluciclatide uptake and perfusion by $\left[{ }^{15} \mathrm{O}\right] \mathrm{H}_{2} \mathrm{O}$ PET.

Methods: Patients with non-small cell lung cancer and melanoma underwent dynamic imaging with arterial sampling following injection of $\left[{ }^{15} \mathrm{O}\right] \mathrm{H}_{2} \mathrm{O}$ and $\left[{ }^{18} \mathrm{~F}\right]$ fluciclatide. Quantification was performed using a one-tissue compartmental model for $\left[{ }^{15} \mathrm{O}\right] \mathrm{H}_{2} \mathrm{O}$ and a two-tissue model for $\left[{ }^{18} \mathrm{~F}\right]$ fluciclatide at volume-of-interest level, and SUV at voxel level.

Results: Tumor binding potential $\left(k_{3} / k_{4}\right.$ ratio) of $\left[{ }^{18} \mathrm{~F}\right]$ fluciclatide tumor was $5.39 \pm 1.46$, consistent with previous studies in breast cancer metastases. Voxel-by-voxel maps of $\left[{ }^{18} \mathrm{~F}\right]$ fluciclatide delivery strongly correlated with $\left[{ }^{15} \mathrm{O}\right] \mathrm{H}_{2} \mathrm{O}-$ based perfusion ( $p<10^{-4}$ tumor, 1,794 $\pm 1,331$ voxels). Interestingly, this correlation was lost when retention of $\left[{ }^{18} \mathrm{~F}\right]$ fluciclatide at late time-points was compared with perfusion ( $p>0.15$ ).
\end{abstract}

Conclusions: Our study suggests tumor $\left[{ }^{18} \mathrm{~F}\right]$ fluciclatide retention is unrelated to tumor perfusion, supporting use of late (60-min) imaging protocols in patients.

Keywords: Fluciclatide; Perfusion; Angiogenesis; PET; Cancer

\section{Background}

Tumor angiogenesis is essential for cancer survival [1]. Many novel therapeutics have been developed which aim to target this process by acting as either angiogenesis inhibitors (e.g. by blocking the action of vascular endothelial growth factor) or as vascular disrupting agents, which target the extracellular matrix [2,3]. Despite the recent advances in therapeutics, there remains a paucity of effective biomarkers which can predict the response of tumors in vivo to these treatments. One area of promise is the development of imaging agents that target the $\alpha v \beta 3$ and $\alpha v \beta 5$ integrins - a set of transmembrane proteins

\footnotetext{
* Correspondence: I.kenny@imperial.ac.uk

${ }^{1}$ Department of Surgery and Cancer, Hammersmith Campus, Imperial College London, London, UK

${ }^{2}$ Department of Medical Oncology, Imperial College Healthcare NHS Trust, London, UK

Full list of author information is available at the end of the article
}

essential for maintaining the relationship between the cell and the extracellular matrix and that have been found to be upregulated on tumor vascular neoendothelium $[4,5]$.

$\left[{ }^{18} \mathrm{~F}\right]$ fluciclatide is an arginine-glycine-aspartic acid (RGD) peptide which binds the $\alpha v \beta 3$ and $\alpha v \beta 5$ integrins with high affinity [6]. We have previously studied the dosimetry and biodistribution of this compound labelled with F-18 in healthy volunteers with positron emission tomography (PET) [7] and the uptake profile of the tracer in patients with metastatic breast cancer [8]. The latter study showed significantly higher radiotracer uptake in lung metastases from breast cancer compared to normal lung tissue. $\left[{ }^{18} \mathrm{~F}\right]$ galacto-RGD is a similar promising agent for studying integrin-related angiogenesis developed by Wester's group [9], and subsequently studied by Beer et al. who demonstrated that there is high uptake of this compound in a variety of 
cancers including melanoma [10] and that uptake correlates with expression of the $\alpha v \beta 3$ integrin measured using immunohistochemistry [11].

The purpose of this study was to understand the relationship between perfusion assessed by $\left[{ }^{15} \mathrm{O}\right] \mathrm{H}_{2} \mathrm{O}$ and ${ }^{18} \mathrm{~F}$-fluciclatide kinetics in non-small cell lung cancer (NSCLC) and melanoma, where Phase 1 trials of humanized antibodies and peptides targeted to integrins have shown promise $[12,13]$. These two tumor types have also been shown to have varying degrees of $\alpha \mathrm{v} \beta 3$ and $\alpha v \beta 5$ expression on tumor cells relative to vascular endothelium with melanoma suggested to have expression on both tumor and vascular endothelium and NSCLC suggested to have expression predominantly on the vascular endothelium [4]. The underlying hypothesis for the study was that fluciclatide retention in tumors is initially dependent on perfursion, but at later timepoints, retention is independent of perfusion.

\section{Methods}

Patients were recruited from oncology clinics at the Imperial College NHS Healthcare trust and the Royal Marsden Hospital NHS Trust. The study was approved by the hospital Local Research Ethics Committee in accordance with the Helsinki Declaration revised guidelines (2008). The eligibility criteria were as follows: patients aged $\leq 80$ years old with a histological proven NSCLC or melanoma, and one site of measureable disease of $\geq 2$-cm diameter, with a treatment-free interval of 3 weeks (or 4 weeks for radiotherapy to the imaging site), life expectancy of at least 3 months, haemoglobin $\leq 10 \mathrm{~g} / \mathrm{dL}$, granulocyte count of $\geq 1.5 \times 10^{9}$, platelet count of $\geq 100 \times 10^{9}$, adequate hepatic function; exclusion criteria: pregnant or lactating patients, sexually active patients who are not employing adequate contraception. Additional details are provided in Table 1.

\section{Radiosynthesis of ${ }^{18} \mathrm{~F}$-fluciclactide and PET scanning procedure}

The chemical synthesis of the precursor of fluciclatide has previously been described [6], radiolabelling was performed at Hammersmith Imanet as previously described on an

Table 1 Demographics of the patients studied

\begin{tabular}{lllll}
\hline Patient & Age & Diagnosis & Stage & Dynamic imaging region \\
\hline 1 & 81 & Melanoma & IV & Thorax \\
2 & 62 & NSCLC & IIIA & Thorax \\
3 & 57 & NSCLC & IIIB & Thorax/Neck \\
4 & 59 & NSCLC & IB & Thorax \\
5 & 66 & NSCLC & IV & Thorax \\
6 & 79 & Melanoma & IV & Knee \\
7 & 59 & NSCLC & IV & Thorax \\
\hline
\end{tabular}

NSCLC, non-small cell lung cancer. automated module (TRACERlab FX F-N; GE Healthcare). The specific activity and radiochemical purity of the injectate, determined by high-performance liquid chromatography (HPLC), was $320 \mathrm{GBq} / \mu \mathrm{mol}$ and $99.7 \%$, respectively.

All patients were scanned on a PET-CT (GE-Discovery $\mathrm{RX}$ ) scanner after being positioned such that the field of view (FOV) included the tumor volume of interest. The axial FOV of the scanner was $15.7 \mathrm{~cm}$ and the transaxial FOV was $70 \mathrm{~cm}$. A low-dose CT scan $(20 \mathrm{~mA}$ and $120 \mathrm{kV})$ was used for attenuation correction prior to the PET scan. A target dose of $600 \mathrm{MBq}\left[{ }^{15} \mathrm{O}\right] \mathrm{H}_{2} \mathrm{O}$ was injected i.v. over $20 \mathrm{~s}$ followed by a 30-s flush with normal saline. Dynamic PET scanning was done for $8 \mathrm{~min}$ and $40 \mathrm{~s}$ with the following frame durations: $30 \mathrm{~s} \times 1,20 \mathrm{~s} \times 1,5 \mathrm{~s} \times 22,10 \mathrm{~s} \times 3$, $30 \mathrm{~s} \times 5$, and $60 \mathrm{~s} \times 3$. After a 2 -min break to allow for radioactive decay $\left({ }^{15} \mathrm{O}\right.$ half-life $\left.=2.04 \mathrm{~min}\right)$, the $\left[{ }^{18} \mathrm{~F}\right]$ fluciclatide scan was performed. $\left[{ }^{18} \mathrm{~F}\right]$ fluciclatide was administered by a bolus i.v. injection over 10 to 30 s. The PET acquisition was conducted as a single bed position dynamic scan (three-dimensional acquisition) centred on the tumor volume of interest (VOI) for $66.5 \mathrm{~min}$. Data were binned into the following time frames: $10 \mathrm{~s} \times 10,20 \mathrm{~s} \times 4,30 \mathrm{~s} \times 4$, $60 \mathrm{~s} \times 7,120 \mathrm{~s} \times 4,300 \mathrm{~s} \times 3$, and $600 \mathrm{~s} \times 3$. Sinograms were Fourier-rebinned into two-dimensional slices and reconstructed (with correction for attenuation, scatter, and dead time) using filtered back-projection (ramp filter kernel full-width-at-half-maximum of $2.0 \mathrm{~mm}$ ). The final images had $128 \times 128 \times 47$ voxels of $2.62 \times 2.62 \times 2.42 \mathrm{~mm}^{3}$.

In order to derive an input function, blood samples were taken during the $\left[{ }^{15} \mathrm{O}\right] \mathrm{H}_{2} \mathrm{O}$ scan via a radial artery cannula continuously at a rate of $5 \mathrm{~mL} / \mathrm{min}$ for the first $9 \mathrm{~min} 40 \mathrm{~s}$; discrete samples were taken at 3,6, and $8 \mathrm{~min}$ $40 \mathrm{~s}$. Similarly, continuous arterial sampling $(5 \mathrm{~mL} / \mathrm{min})$ was performed for $10 \mathrm{~min}$ during the $\left[{ }^{18} \mathrm{~F}\right]$ fluciclatide scan; seven discrete samples ( 5 to $10 \mathrm{~mL}$ ) were taken at $2.5,5,10,15,30,45,60 \mathrm{~min}$. Metabolite analyses were performed on the 2.5-, 5-, 10-, 30-, and 60-min samples. The computation of $\left[{ }^{18} \mathrm{~F}\right]$ fluciclatide parent fraction and input function was carried out as described in [7].

\section{Data analysis}

Volumes-of-interest (VOIs) were manually defined around visible tumors from the $\left[{ }^{18} \mathrm{~F}\right]$ fluciclatide summed images using the Analyze software (Version 7). The same VOIs were applied to the $\left[{ }^{15} \mathrm{O}\right] \mathrm{H}_{2} \mathrm{O}$ scan to extract tumor time-activity curves (TACs) for both tracers.

To estimate the perfusion from the $\left[{ }^{15} \mathrm{O}\right] \mathrm{H}_{2} \mathrm{O}$ data, a one-tissue compartment model was employed:

$$
C_{\mathrm{T}}(t)=\left(1-V_{\mathrm{b}}\right) \times\left[K_{1} \times\left[C_{\mathrm{a}}(t) \otimes \exp \left(-k_{2} \times t\right)\right]+V_{\mathrm{b}} C_{\mathrm{a}}(t)\right.
$$

In Equation 1, $C_{\mathrm{T}}(t)$ indicates the tumor TAC, $C_{\mathrm{a}}(t)$ the measured radioactivity concentration in arterial 
blood, $K_{1}$ the regional blood flow $\left(\mathrm{mL} / \mathrm{cm}^{3} / \mathrm{min}\right), k 2$ $(1 / \mathrm{min})$ the transfer rate from tissue to blood, $V_{\mathrm{b}}$ (unitless) the blood volume fraction and $\otimes$ the convolution operator. $K_{1}, k_{2}$, and $V_{\mathrm{b}}$ were estimated for each VOI by fitting $C_{\mathrm{T}}(t)$ to Equation 1 using the standard weighted non-linear least squares (WNLLS). WNLLS minimizes the weighted residual sum of squares (WRSS)

$$
\operatorname{WRSS}(p)=\sum_{i=1}^{n} w_{i}\left[C\left(t_{i}, p\right)^{M O D E L}-C_{T}\left(t_{i}\right)\right]^{2}
$$

with $p, t_{i}$, and $n$ indicating respectively the parameter vector $\left[K_{1} ; k_{2} ; V_{\mathrm{b}}\right]$, the mid-time of the $i$ th frame, and the number of frames. In Equation 2 weights wi were set to

$$
w_{i}=\frac{\Delta_{i}}{C\left(t_{i}\right) \exp \left(\lambda t_{i}\right)}
$$

Tomasi G 2009, [14] with $\Delta_{i}$ and $\lambda$ indicating respectively the duration of the $i$ th frame and the half-life of ${ }^{15} \mathrm{O}$. The delay between $C_{\mathrm{a}}(t)$ and $C_{\mathrm{T}}(t)$ was modelled by shifting $C_{\mathrm{T}}(t)$ of $i$ seconds $(i=0,1, \ldots, 30)$ and then retaining the value of the delay which gave rise to the smallest WRSS.

A two-tissue reversible compartment model was used to model $\left[{ }^{18} \mathrm{~F}\right]$ fluciclatide kinetics, consistent with our previous study [8]. The metabolite-corrected measured arterial concentration of $\left[{ }^{18} \mathrm{~F}\right]$ fluciclatide was used as input function for estimating with WNNLS the parameters $K_{1}\left(\mathrm{~mL} / \mathrm{cm}^{3} / \mathrm{min}\right), k_{2}(1 / \mathrm{min}), k_{3}(1 / \mathrm{min}), k_{4}(1 / \mathrm{min}), V_{\mathrm{b}}$ (unitless) for each VOI. Weights were computed from Equation 3. The parameter $k_{3} / k_{4}$ ratio - a measure of the binding potential - was then computed for each VOI; as we previously reported [8], this parameter was the best of a number of parameters examined to differentiate between healthy and tumor VOIs.

$K_{1}, k_{2}$, and $V_{\mathrm{b}}$ were also estimated at the voxel level for $\left[{ }^{15} \mathrm{O}\right] \mathrm{H}_{2} \mathrm{O}$ using the standard Basis Function Method approach [15]. The final maps, however, were extremely noisy because of the high noise of $\left[{ }^{15} \mathrm{O}\right] \mathrm{H}_{2} \mathrm{O}$ data and we did not include them in the analysis. Parametric maps were not generated in the case of $\left[{ }^{18} \mathrm{~F}\right]$ fluciclatide because of the difficulty of fitting a five-parameter model at the voxel level. To analyze the correlation between perfusion and $\left[{ }^{18} \mathrm{~F}\right]$ fluciclatide uptake at the voxel level we employed the following semi-quantitative parameters. For $\left[{ }^{15} \mathrm{O}\right] \mathrm{H}_{2} \mathrm{O}$ we used

$$
S U V_{\text {water }}\left[m^{2} / L\right]=\frac{\text { mean }\left[C_{T}(t)_{0-1.5 \mathrm{~min}}\right]}{\frac{\text { Injected dose }}{B S A}}
$$

In Equation 4, we arbitrarily chose $1.5 \mathrm{~min}$ as the final time, instead of the scan duration of $8 \mathrm{~min}$, to define a parameter describing $\left[{ }^{15} \mathrm{O}\right] \mathrm{H}_{2} \mathrm{O}$ delivery (i.e. flow) before washout took place. To measure $\left[{ }^{18} \mathrm{~F}\right]$ fluciclatide retention, we employed

$$
S U V_{\text {fluci }} \text { RETENTION }\left[\mathrm{m}^{2} / L\right]=\frac{C_{T}\left(t_{i}\right)}{\frac{\text { Injected dose }}{B S A}}
$$

In Equations 4 and 5, BSA denotes the body surface area, and $t_{i}$ in Equation 5 indicates the mid-time of the last frame of the $\left[{ }^{18} \mathrm{~F}\right]$ fluciclatide scan $(60.5 \mathrm{~min})$. We also defined a parameter describing $\left[{ }^{18} \mathrm{~F}\right]$ fluciclatide delivery as

$$
S U V_{\text {fluci DELIVERY }}\left[\mathrm{m}^{2} / L\right]=\frac{\text { mean }\left[C_{T}(t)_{0-1.5 \mathrm{~min}}\right]}{\frac{\text { Injected dose }}{B S A}}
$$

where the same upper limit used for SUVwater was employed. These parameters were computed for each voxel of the tumor VOIs and the agreement of SUVwater with SUVfluci RETENTION and SUVfluci DELIVERY was assessed using Spearman's correlation coefficient.

\section{Results}

The activity of injected $\left[{ }^{18} \mathrm{~F}\right]$ fluciclatide ranged between 290 and $383 \mathrm{MBq}$, and that of $\left[{ }^{15} \mathrm{O}\right] \mathrm{H}_{2} \mathrm{O}$ between 448 and $697 \mathrm{MBq}$. Due to tracer failure and patient comfort reasons, PET scans of both tracers were carried out in four patients; for two patients, only the $\left[{ }^{18} \mathrm{~F}\right]$ fluciclatide data were obtained, and for the remaining patient, only the $\left[{ }^{15} \mathrm{O}\right] \mathrm{H}_{2} \mathrm{O}$ data were obtained.

Typical images of $\left[{ }^{15} \mathrm{O}\right] \mathrm{H}_{2} \mathrm{O}$ and $\left[{ }^{18} \mathrm{~F}\right]$ fluciclatide are shown in Figure $1 \mathrm{~A}, \mathrm{~B}$, respectively. The quality of the fits was good for both tracers. Examples are displayed in Figure 2A for $\left[{ }^{15} \mathrm{O}\right] \mathrm{H}_{2} \mathrm{O}$ and Figure $2 \mathrm{~B}$ for $\left[{ }^{18} \mathrm{~F}\right]$ fluciclatide. In Table 2, the kinetic parameters for $\left[{ }^{15} \mathrm{O}\right] \mathrm{H}_{2} \mathrm{O}$ and the $k_{3} / k_{4}$ ratios of $\left[{ }^{18} \mathrm{~F}\right]$ fluciclatide at the VOI level are reported. For completeness, we also reported SUV parameters for $\left[{ }^{18} \mathrm{~F}\right]$ fluciclatide.

The $k_{3} / k_{4}$ ratio of $\left[{ }^{18} \mathrm{~F}\right]$ fluciclatide was $5.39 \pm 1.46$, in good agreement with our previous results in lung metastases $\left(k_{3} / k_{4}=6.09 \pm 3.04\right)$. There was a good correlation (Pearson $r=0.71, p$ value $=0.06$, Figure 3 ) between SUVfluci RETENTION ( $x$-axis) and $k_{3} / k_{4}(y$-axis) at the VOI level supporting the use of the semi-quantitative parameter SUVfluci RETENTION as a measure of retention.

To investigate the relationship between perfusion and $\left[{ }^{18} \mathrm{~F}\right]$ fluciclatide retention, which was not feasible at the VOI level due to the low number of subjects completing both scans, we compared SUVwater with SUVfluci RETENTION or SUVfluci DELIVERY at the voxel level. As expected, SUVwater was well correlated with SUVfluci DELIVERY, the correlation being statistically significant for voxels within all four tumors ( $p$ value $<10^{-4}$ for all VOIs). The correlation between SUVwater and SUVfluci RETENTION, on the other hand, was not statistically 

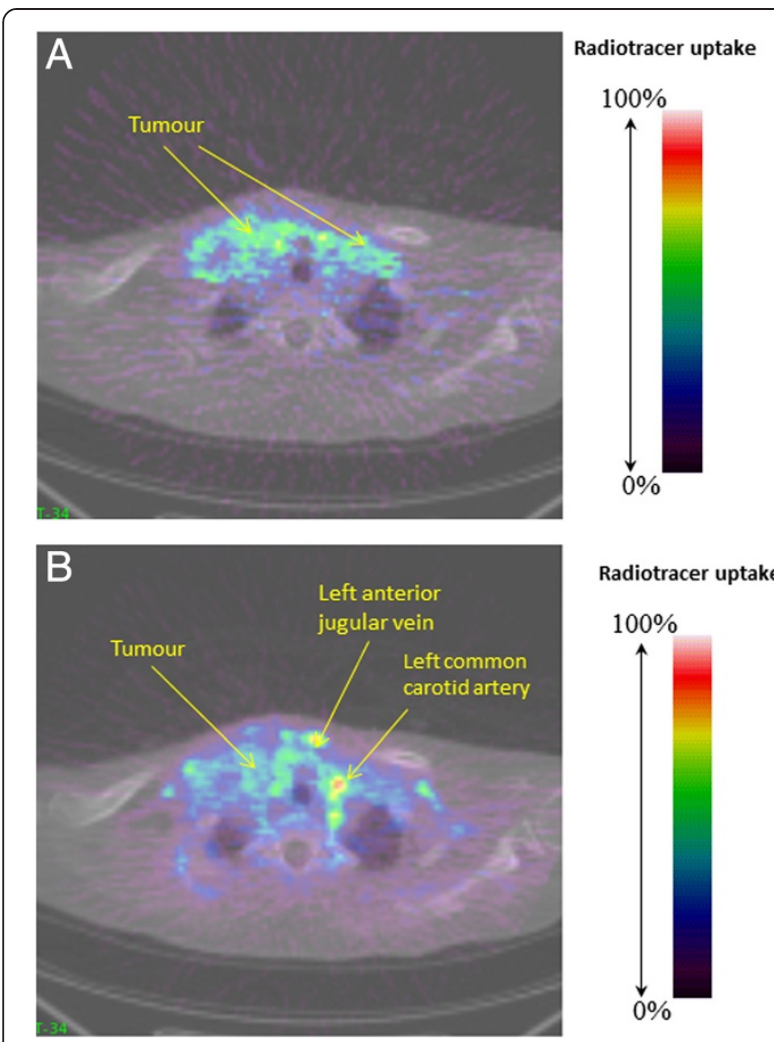

Radiotracer uptake

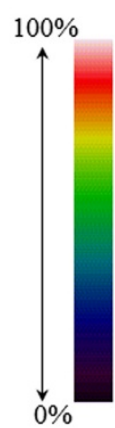

Figure 1 Summed images of $(A)\left[{ }^{15} \mathrm{O}_{\mathrm{H}} \mathrm{O}\right.$ and (B) $\left[{ }^{18} \mathrm{~F}\right]$ fluciclatide. These were in a patient with non-small cell lung cancer with bilateral cervical lymph node metastases. The blood vessels are labelled.
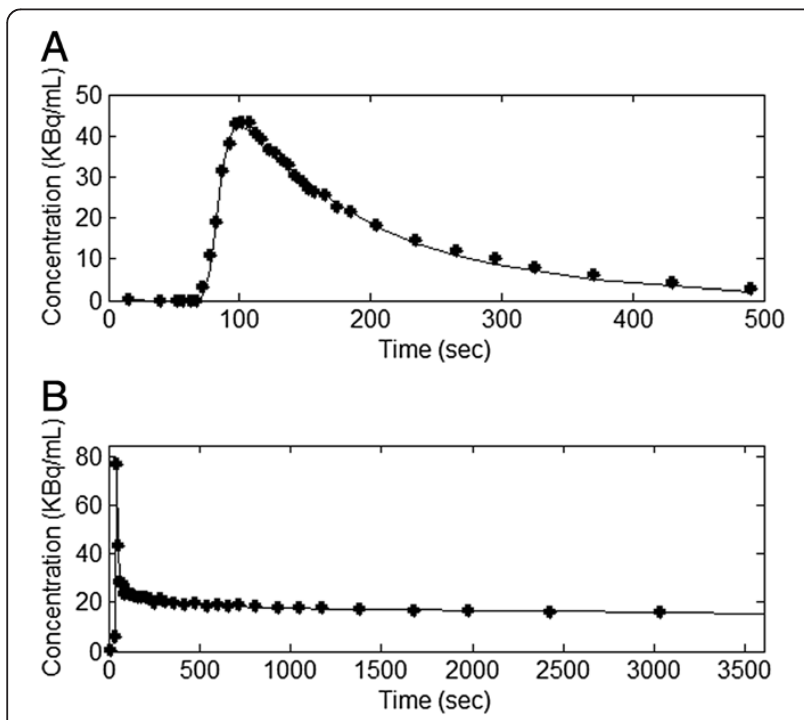

Figure 2 Time-activity curves for $(A)\left[{ }^{15} \mathrm{O}^{\mathrm{H}} \mathrm{H}_{2} \mathrm{O}\right.$ and (B) $\left[{ }^{18}\right.$ F fluciclatide.
Table 2 Kinetic parameters for $\left[{ }^{15} \mathrm{O}\right] \mathrm{H}_{2} \mathrm{O}$ and $\left[{ }^{18} \mathrm{~F}\right]$ fluciclatide at the VOI level

\begin{tabular}{|c|c|c|c|c|c|}
\hline $\begin{array}{l}\text { Patient } \\
\text { number }\end{array}$ & $\begin{array}{l}K_{1}\left[{ }^{15} \mathrm{O}\right] \\
\mathrm{H}_{2} \mathrm{O} \\
\left(\mathrm{mL} / \mathrm{cm}^{3} / \mathrm{min}\right)\end{array}$ & $\begin{array}{l}k_{2}\left[{ }^{15} \mathrm{O}\right] \\
\mathrm{H}_{2} \mathrm{O} \\
(\mathrm{L} / \mathrm{min})\end{array}$ & $\begin{array}{l}k_{3} / k_{4}\left[{ }^{18} \mathrm{~F}\right] \\
\text { fluciclatide } \\
\text { (unitless) }\end{array}$ & $\begin{array}{l}\text { SUVfluci } \\
\text { DELIVERY } \\
\left(\mathrm{m}^{2} / \mathrm{L}\right)\end{array}$ & $\begin{array}{l}\text { SUVfluci } \\
\text { RETENTION } \\
\left(\mathrm{m}^{2} / \mathrm{L}\right)\end{array}$ \\
\hline 1 & 0.15 & 0.32 & 4.34 & 0.070 & 0.049 \\
\hline 2 & 0.16 & 0.38 & 4.37 & 0.137 & 0.072 \\
\hline 3 & 0.38 & 0.61 & 4.87 & 0.045 & 0.064 \\
\hline 4 & N/A & N/A & 7.81 & 0.050 & 0.087 \\
\hline 5 & N/A & N/A & 6.57 & 0.040 & 0.070 \\
\hline 6 & 0.68 & 0.88 & N/A & N/A & N/A \\
\hline \multicolumn{6}{|l|}{ (Tumor\#1) } \\
\hline 6 & 0.39 & 0.62 & N/A & N/A & $\mathrm{N} / \mathrm{A}$ \\
\hline \multicolumn{6}{|l|}{ (Tumor\#2) } \\
\hline 7 & 0.44 & 0.72 & 4.38 & 0.077 & 0.062 \\
\hline
\end{tabular}

significant (Spearman $p$ value $>0.15$ for all tumors). This information is displayed in Figure 4, which shows the relationship between SUVwater ( $x$-axis) and SUVfluci DELIVERY ( $y$-axis, Figure 4A,C,E,G for patients 1, 2, 3, and 7, respectively) and between SUVwater and SUVfluci RETENTION ( $y$-axis, Figure 4B,D,F,H). The plots on the same row correspond to the same patients and the continuous line is the fitted line of equation $y=m x+q$. Whereas a monotonic relationship can be noticed between SUVwater and SUVfluci DELIVERY, there is no clear visual relationship between SUVwater and SUVfluci RETENTION .

\section{Discussion}

There is an increasing need for effective biomarkers which can reliably predict the response to novel therapeutics acting as angiogenesis inhibitors or as vascular disrupting

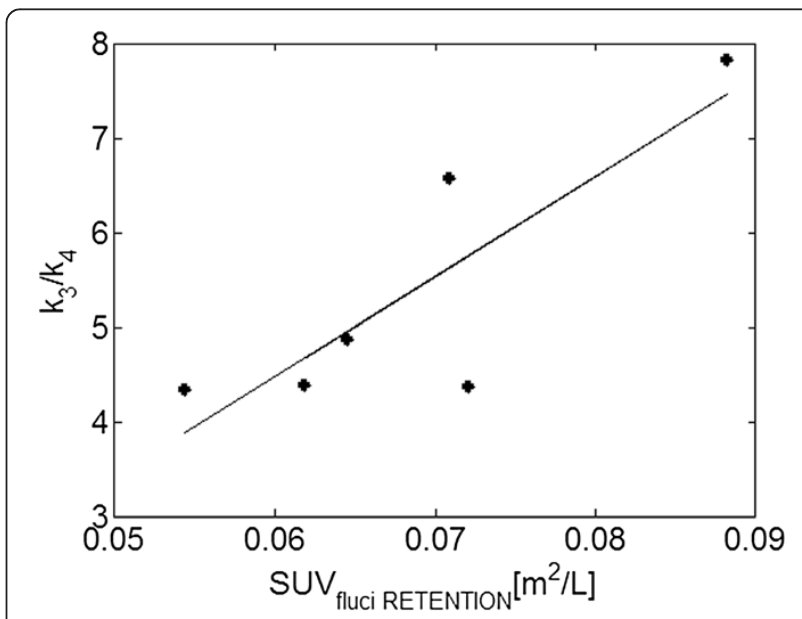

Figure 3 Correlation between $\left[{ }^{18} \mathrm{~F}\right]$ fluciclatide retention SUV and $k_{3} / k_{4}$ ratio. 


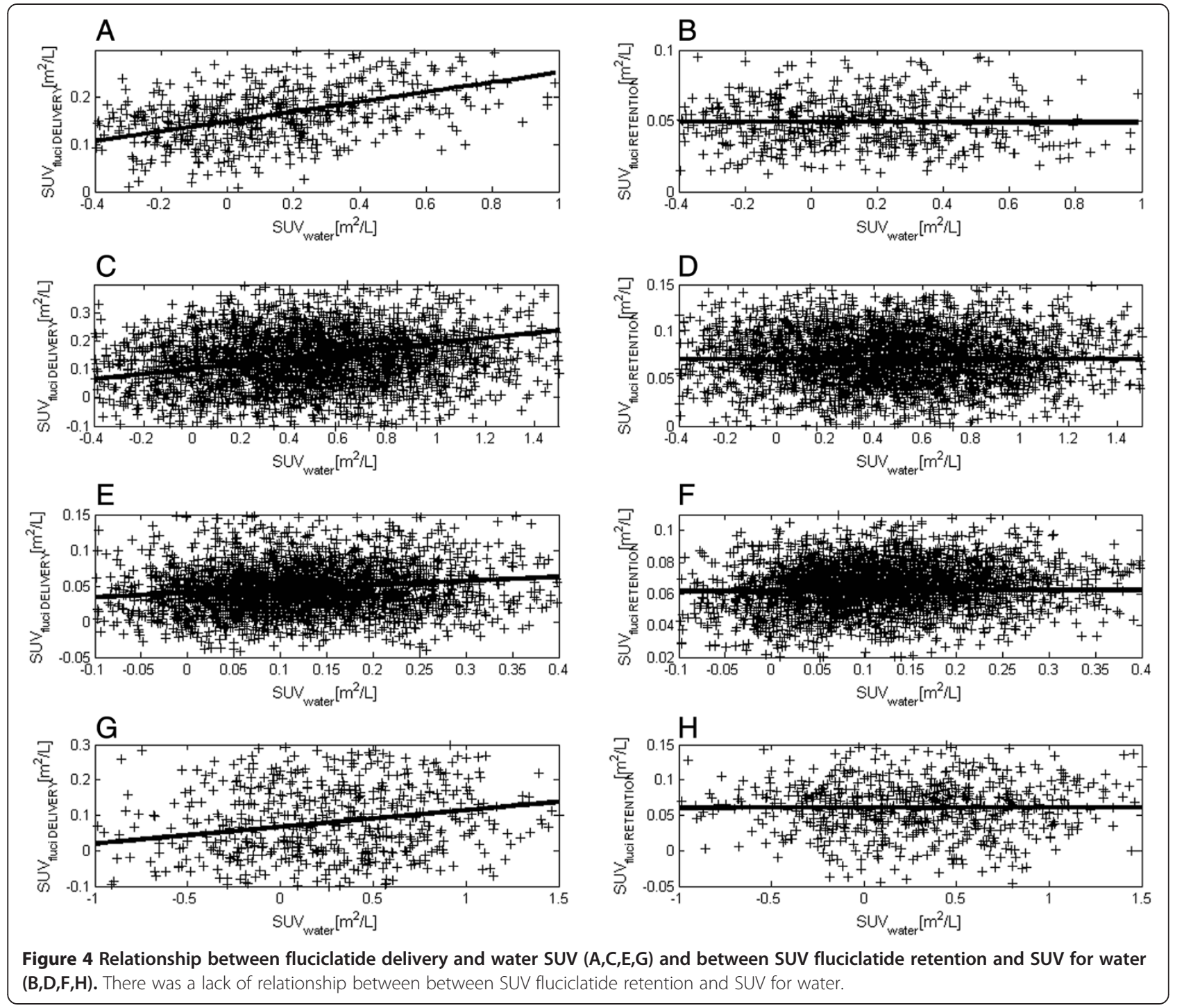

agents in vivo, and the RGD-based ligand, $\left[{ }^{18} \mathrm{~F}\right]$ fluciclatide, is very promising in this respect. Given the haemodynamic context of angiogenesis, the uptake characteristics of a good biomarker should be independent of blood flow. Consequently, the objective of this study, which is of scientific relevance to the use of this and possibly other RGD-based ligands, was to assess the dependency of $\left[{ }^{18} \mathrm{~F}\right]$ fluciclatide uptake on perfusion. The major finding of the study is that, at the voxel level, $\left[{ }^{18} \mathrm{~F}\right]$ fluciclatide retention is not correlated with perfusion measured by $\left[{ }^{15} \mathrm{O}\right] \mathrm{H}_{2} \mathrm{O}$ uptake. We contrast this finding with a good agreement, as expected, between $\left[{ }^{15} \mathrm{O}\right] \mathrm{H}_{2} \mathrm{O}$ uptake and $\left[{ }^{18} \mathrm{~F}\right]$ fluciclatide delivery suggesting that the lack of correlation between $\left[{ }^{15} \mathrm{O}\right] \mathrm{H}_{2} \mathrm{O}$ uptake and $\left[{ }^{18} \mathrm{~F}\right]$ fluciclatide retention was not due to noise. Incidentally, the two-tissue compartment model which we validated for $\left[{ }^{18} \mathrm{~F}\right]$ fluciclatide in our previous report proved to be appropriate also for this dataset, and the mean $k_{3} / k_{4}$ ratio obtained in this study
$(5.39 \pm 1.46)$ was in good agreement with the results obtained in lung metastases $\left(k_{3} / k_{4}=6.09 \pm 3.04\right)$.

The main limit in this initial study is the small sample size, as only four out of seven patients successfully completed both scans, which hampered the quantification of the relationship between $K_{1}$ of $\left[{ }^{15} \mathrm{O}\right] \mathrm{H}_{2} \mathrm{O}$ and $\left[{ }^{18} \mathrm{~F}\right]$ fluciclatide $k_{3} / k_{4}$ at the VOI level. In this regard our future plan is to test further these preliminary findings on a larger cohort of subjects and also to apply/develop appropriate noise-reduction approaches which will allow us to compare $K_{1}$ maps of $\left[{ }^{15} \mathrm{O}\right] \mathrm{H}_{2} \mathrm{O}$ and $k_{3} / k_{4}$ of $\left[{ }^{18} \mathrm{~F}\right]$ fluciclatide at the voxel level.

\section{Conclusion}

In conclusion, these preliminary results support the view that $\left[{ }^{18} \mathrm{~F}\right]$ fluciclatide binding (retention) is not dependent on perfusion, highlighting the potential of this radiotracer in the study of receptors expressed on the 
neovasculature. The study supports the use of late imaging protocols (60 min post injection) for assessment of $\left[{ }^{18} \mathrm{~F}\right]$ fluciclatide uptake.

\section{Abbreviations}

BSA: Body surface area; HPLC: High-performance liquid chromatography; NSCLC: Non-small cell lung cancer; RGD: Arginine-glycine-aspartame; SUV: Standardised uptake value; VOI: Volume of interest; WNLSS: Weighted non-linear least squares; WRSS: Weighted residual sum of squares.

\section{Competing interests}

The authors declare that they have no competing interests.

\section{Authors' contributions}

LK contributed to the study design, data acquisition and analysis, manuscript writing and review. GT contributed to the data analysis, manuscript writing and review. CB, JL, MG and SM contributed to the study design and manuscript review. FT and EO contributed to the study design, manuscript writing, and critical review. All authors read and approved the final manuscript.

\section{Acknowledgements}

This study was funded by the MRC (programme grant MC_A652_5PY80), Experimental Cancer Medicine Centre grant C37/A7283, and an NIHR clinician scientist fellowship grant (LKenny 09/009) and supported by the NIHR Cancer Clinical Research Network UK. We wish to thank Kasia Kozlowski and Laura Maher, the radiographers and staff of Imanet and GE Healthcare for their assistance with this project. This study was supported by NIHR Cancer Clinical Research Network.

\section{Author details}

'Department of Surgery and Cancer, Hammersmith Campus, Imperial College London, London, UK. ${ }^{2}$ Department of Medical Oncology, Imperial College Healthcare NHS Trust, London, UK. ${ }^{3}$ Centre for Neuroimaging, Institute of Psychiatry, King's College London, London, UK. ${ }^{4}$ Royal Marsden NHS Trust, London, UK.

Received: 8 April 2014 Accepted: 20 May 2014

Published: 8 August 2014

\section{References}

1. Carmeliet P, Jain RK: Molecular mechanisms and clinical applications of angiogenesis. Nature 2011, 473:298-307.

2. Daenen $L G$, Roodhart JM, Shaked $Y$, Voest EE: Vascular disrupting agents (VDAs) in anticancer therapy. Curr Clin Pharmacol 2010, 5:178-185.

3. Sleijfer S, Ray-Coquard I, Papai Z, Le Cesne A, Scurr M, Schoffski P, Collin F, Pandite L, Marreaud S, De Brauwer A, van Glabbeke M, Verweij J, Blay JY: Pazopanib, a multikinase angiogenesis inhibitor, in patients with relapsed or refractory advanced soft tissue sarcoma: a phase II study from the European organisation for research and treatment of cancer-soft tissue and bone sarcoma group (EORTC study 62043). J Clin Oncol 2009, 27:3126-3132.

4. Weis SM, Cheresh DA: alphav Integrins in Angiogenesis and Cancer. Cold Spring Harb Perspect Med 2011, 1:a006478.

5. Avraamides CJ, Garmy-Susini B, Varner JA: Integrins in angiogenesis and lymphangiogenesis. Nat Rev Cancer 2008, 8:604-617.

6. Indrevoll B, Kindberg GM, Solbakken M, Bjurgert E, Johansen JH, Karlsen H, Mendizabal M, Cuthbertson A: NC-100717: a versatile RGD peptide scaffold for angiogenesis imaging. Bioorg Med Chem Lett 2006, 16:6190-6193.

7. Kenny LM, Coombes RC, Oulie I, Contractor KB, Miller M, Spinks TJ, McParland B, Cohen PS, Hui AM, Palmieri C, Osman S, Glaser M, Turton D, Al-Nahhas A, Aboagye EO: Phase I trial of the positron-emitting Arg-Gly-Asp (RGD) peptide radioligand ${ }^{18} \mathrm{~F}-\mathrm{AH} 111585$ in breast cancer patients. J NuCl Med 2008, 49:879-886.

8. Tomasi G, Kenny L, Mauri F, Turkheimer F, Aboagye EO: Quantification of receptor-ligand binding with $\left[{ }^{18} \mathrm{~F}\right]$ fluciclatide in metastatic breast cancer patients. Eur J Nucl Med Mol Imaging 2011, 38(12):2186-2197.

9. Haubner R, Kuhnast B, Mang C, Weber WA, Kessler H, Wester HJ, Schwaiger M: $\left[{ }^{18} \mathrm{~F}\right]$ Galacto-RGD: synthesis, radiolabeling, metabolic stability, and radiation dose estimates. Bioconjug Chem 2004, 15:61-69.
10. Beer AJ, Haubner R, Goebel M, Luderschmidt S, Spilker ME, Wester HJ, Weber WA, Schwaiger M: Biodistribution and pharmacokinetics of the alphavbeta3-selective tracer ${ }^{18} \mathrm{~F}$-galacto-RGD in cancer patients. J Nucl Med 2005, 46:1333-1341.

11. Beer AJ, Haubner R, Sarbia M, Goebel M, Luderschmidt S, Grosu AL, Schnell $\mathrm{O}$, Niemeyer M, Kessler H, Wester HJ, Weber WA, Schwaiger M, Colevas AD, Grossman SA: Positron emission tomography using $\left[{ }^{18} \mathrm{~F}\right]$ Galacto-RGD identifies the level of integrin alpha(v)beta3 expression in man. Clin Cancer Res 2006, 12:3942-3949.

12. Nabors LB, Mikkelsen T, Rosenfeld SS, Hochberg F, Akella NS, Fisher JD, Cloud GA, Zhang Y, Carson K, Wittemer SM, et al: Phase I and correlative biology study of cilengitide in patients with recurrent malignant glioma. J Clin Oncol 2007, 25:1651-1657.

13. Albert JM, Cao C, Geng L, Leavitt L, Hallahan DE, Lu B: Integrin alpha v beta 3 antagonist Cilengitide enhances efficacy of radiotherapy in endothelial cell and non-small-cell lung cancer models. Int J Radiat Oncol Biol Phys 2006, 65:1536-1543.

14. Tomasi G, Bertoldo A, Bishu S, Unterman A, Smith CB, Schmidt KC: Voxel-based estimation of kinetic model parameters of the L-[1- $\left.{ }^{11} \mathrm{C}\right]$ leucine PET method for determination of regional rates of cerebral protein synthesis: validation and comparison with region-of-interest-based methods. J Cereb Blood Flow Metab 2009, 29:1317-1331

15. Gunn RN, Lammertsma AA, Hume SP, Cunningham VJ: Parametric imaging of ligand-receptor binding in PET using a simplified reference region model. Neuroimage 1997, 6:279-287.

\section{doi:10.1186/s13550-014-0030-x}

Cite this article as: Kenny et al:: Preliminary clinical assessment of the relationship between tumor alphavbeta3 integrin and perfusion in patients studied with $\left[{ }^{18} \mathrm{~F}\right]$ fluciclatide kinetics and $\left[{ }^{15} \mathrm{O}\right] \mathrm{H}_{2} \mathrm{O}$ PET. EJNMMI Research 2014 4:30

\section{Submit your manuscript to a SpringerOpen ${ }^{\mathcal{D}}$ journal and benefit from:}

- Convenient online submission

- Rigorous peer review

- Immediate publication on acceptance

- Open access: articles freely available online

- High visibility within the field

- Retaining the copyright to your article

Submit your next manuscript at $>$ springeropen.com 